\title{
Proximity effect and strong coupling superconductivity in nanostructures built with an STM
}

\author{
H. Suderow ${ }^{1}$, E. Bascones ${ }^{2,3}$, A. Izquierdo ${ }^{1}$, F. Guinea ${ }^{2}$, S. Vieira ${ }^{1}$ \\ ${ }^{1}$ Laboratorio de Bajas Temperaturas*, Departamento de Fisica de la Materia Condensada, Instituto de Ciencia de Materiales \\ Nicolás Cabrera, Universidad Autonoma de Madrid. E-28049 Madrid. Spain \\ ${ }^{2}$ Instituto de Ciencia de Materiales de Madrid ${ }^{*}$, Consejo Superior de Investigaciones Cientificas, Campus de Cantoblanco. \\ E-28049 Madrid-Spain \\ ${ }^{3}$ Department of Physics, The University of Texas at Austin, Austin, Texas 78712
}

(November 5, 2018)

\begin{abstract}
We present high resolution tunneling spectroscopy data at very low temperatures on superconducting nanostructures of lead built with an STM. By applying magnetic fields, superconductivity is restricted to length scales of the order of the coherence length. We measure the tunneling conductance and analyze the phonon structure and the low energy DOS. We demonstrate the influence of the geometry of the system on the magnetic field dependence of the tunneling density of states, which is gapless in a large range of fields. The behavior of the features in the tunneling conductance associated to phonon modes are explained within current models.
\end{abstract}

PACS numbers: 74.50.+r, 74.80.Fp

During the last years there has been an increasing interest on the understanding of the physical properties of the nanoscopic size objects. This has been promoted by the rapid development of new experimental tools that permit a direct access to the realm of the nanoworld. One of the most widely known is the scanning tunneling microscope (STM) [1], which permits the creation of metallic nanostructures that can be at the same time imaged and characterized in situ [2]3]. STM operation at low temperatures has produced new and interesting results, many of them in the field of superconductivity [4.

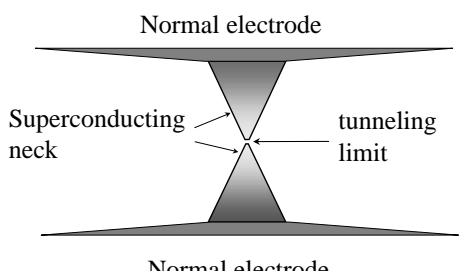

FIG. 1. We consider a connecting neck, formed according to the method described elsewhere [2 3, 9 11], broken into two parts to be able to measure the tunneling density of states. The diagram is a sketch to show the geometry that we use to make a simplified model of the nanostructure built with this procedure.

Recently, and using a low temperature STM, we have studied transport through nanosized metallic necks [9, 10]. In the case of lead we have shown that it is possible to form a neck of a few hundreds of nanometer length [2,3] which connects the two bulk electrodes and which can be described as two opposed truncated cones. Application of an external magnetic field $\mathrm{H}$ at temperatures below the superconducting critical temperature of the bulk creates a very singular nanostructure when $\mathrm{H}$ is higher than bulk critical field, $\mathrm{H}_{c}$ [11]. The bulk electrodes transit to the resistive state, and superconductivity is confined to the neck. The transport properties are governed by the diameter of the smallest cross section, which can be changed in a highly reproducible way from the large point contact to the single atom point contact regimes [9, 10]. A detailed Ginzburg-Landau analysis has been done by the authors of 12. to examine the order parameter in this system.

Here we present new tunneling conductance measurements in these connecting necks as a function of the magnetic field done by breaking the neck in-situ into two parts, after its preparation (described below). This is a singular system with two superconducting "hills" ending in a sharp tip of atomic dimensions (fig.1). As the whole procedure is done at low temperatures (about $0.5 \mathrm{~K}$ ), we can neglect atomic diffusion so that the geometry of the system is not significantly modified when breaking. The conductance is in the tunneling limit and is proportional to the convolution of the densities of states (DOS) of both parts of the broken neck [13]. In this way we gain direct experimental access to the DOS of a nanoscopic structure with applied and fundamental interest. Note that it has the same form as two STM tips, one opposed in front of the other (fig.1). With an in-situ positioning $x-y$ table we can in principle transport one part of the neck to another place to use it as a superconducting STM tip that probes a given sample [14]. This is of major interest because it opens the possibility for new applications as local Josephson spectroscopy or spin-polarized tunneling, which needs a magnetic field 15]17. Other methods have been successfully used to make superconducting STM tips [16,17] but, to our knowledge, no data are available under magnetic fields.

We use a conventional STM set-up in a ${ }^{3} \mathrm{He}$ cryo- 
stat. The sample and tip (both of $\mathrm{Pb}$ ) are cleaned by a mechanical method and mounted on the STM which is cooled down as fast as possible (about five minutes) in order to minimize the formation of oxydes on the surface. The fabrication consists of a series of repeated indentations done in-situ at low temperatures, as described in previous work 2, 3,9 111. The neck is broken to reach the tunneling regime, where the estimated work function is of the order of several $\mathrm{eV}$ [2, 18], indicating that we have a clean vacuum tunnel junction between both parts of the neck. The measurement is done with a tunneling resistance of about $10 M \Omega$. Special care is put on the electronic filtering of the set-up in order to have the maximal energy (voltage) resolution and to avoid artificial smearing of the conductance curves. The resolution of the set-up is of $35 \mu \mathrm{V}$, comparable to the lowest temperature of the sample of $400 \mathrm{mK}$.

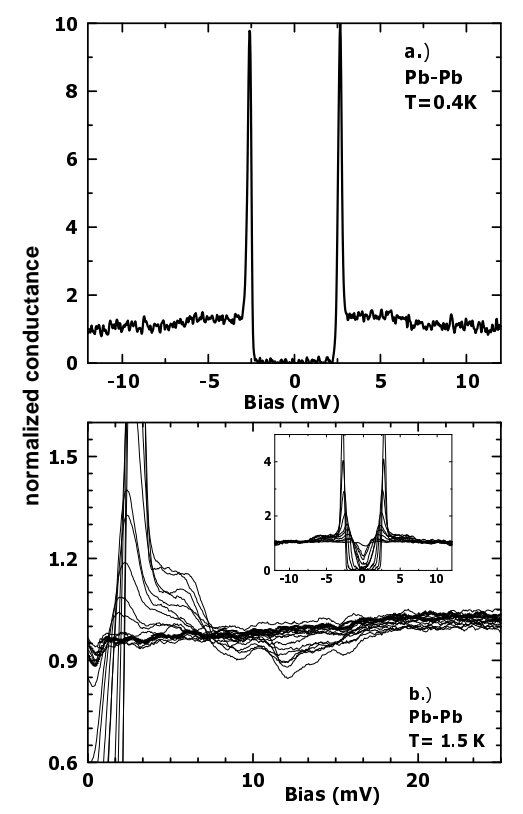

FIG. 2. a.)Conductance as a function of the applied bias as measured with an STM at $0.4 \mathrm{~K}$ with tip and sample of $\mathrm{Pb}$ and zero magnetic field. b.) The phonon structure as a function of the magnetic field (note the change in the axis) at $1.5 \mathrm{~K}$ at fields $0,0.17,0.34,0.51,0.67,0.84,1.01,1.18,1.24$ and $1.46 \mathrm{~T}$ applied perpendicularly to the neck. The inset shows the tunneling conductance in the same units but in a larger range of values.

Figure 2a.) shows the conductance at zero field and $0.5 \mathrm{~K}$. As $\mathrm{Pb}$ is a strong coupling superconductor, the features due to phonon modes are clearly observed in the tunneling conductance 19 (fig.2b). According to the well known properties of strong coupling superconductors, a peak in the effective phonon spectrum $\alpha^{2} F(\omega)$ gives a peak in the voltage derivative of the conductance, located at $\epsilon_{L, T}=2 \Delta_{0}+\omega_{L, T}$ and therefore the features shown in fig.2b. No significant difference neither in the value of the superconducting gap $\Delta_{0}=1.32 \mathrm{mV}$ nor in the phonon modes $\left(\omega_{T}=4.4 \mathrm{mV}\right.$ and $\left.\omega_{L}=8.6 \mathrm{mV}\right)$ with respect to planar junction experiments is found within the experimental resolution (5\%) [13]. The magnetic field for total destruction of the supeconducting correlations depends on the form of the neck 20. The example shown in the figure corresponds to a neck having a critical field of about twenty times the bulk critical field $\left(H_{c}^{P b}(0 K)=0.08 T\right)$. Experiments done with the field applied parallel or perpendicular to the axis of the neck show the same behavior, i.e., the destruction of the superconducting features in the tunneling conductance at magnetic fields much larger than the bulk critical field, depending on the form of the neck 20].

To discuss the results, we first try to consider the known example of thin wires of type I superconductors with lateral dimensions smaller than the London penetration depth, which remain superconducting at magnetic fields much higher than the bulk value [22]. For instance, superconductivity in an infinite cylindrical wire can be described by a single pair breaking parameter given by (in units of $\Delta_{0}$ ) $\Gamma_{0}=\frac{\xi^{2} R^{2}}{3 l_{H}^{4}}$, where $\Delta_{0}$ is the zero field order parameter, $R$ is the radius of the cylinder and $l_{H}$ the magnetic length $l_{H}=256 \AA / \sqrt{H}$. The peak in the DOS is rounded and the gap and order parameter are reduced with respect to the zero field value 22 25. The gap remains finite in a large range of fields, and it is only very close to the critical field (above about $0.95 H_{c}$ ) where gapless superconductivity sets in. In the inset of fig.3a we compare the measured I-V curve with the values of $\Gamma_{0}$ which best fit the experiment. Neither the low energy part, nor the magnetic field dependence are reproduced. The experimental curves show a finite current at low voltages, corresponding to low energy states within the gap, even for fields small compared to the critical one. The density of states at zero voltage is finite already at fields between 20 and $30 \%$ the field for complete disappearance of superconductivity, in clear contradiction with the homogeneous pair breaking model 22].

A better description of the conic, non uniform geometry of the necks leads to a more satisfactory result. In a previous work we proposed [9], in the framework of Usadel equations [26], a model in which the field enters as an effective, position dependent, pair breaking rate. The equations are solved self-consistently, allowing us to obtain a complete description of our system in terms of energy and distance to the center of the broken neck. The main difference with respect to models using a homogeneous geometry 11,22 is that there is a smooth transition to the resistive state as the radius of the neck increases. The density of states calculated at the center of the structure remains finite at low energies due to the proximity effect of the region which is not superconducting, in agreement with the experiment (fig.3). Using parameters compatible with the geometry of the measured 
structure we can get a good fit (fig.3) for both parallel and perpendicular fields using the same set of parameters for the whole series of curves measured in a single structure (see inset of fig.3b, note that not all curves are presented for clarity).

Previous work has also shown that the conic geometry of our system needs to be taken into account to understand experiments done in the single atom point contact limit [9], but in that case, the I-V curves present significant subgap conductance and are less sensitive to details of the density of states. The tunneling conductance data however give a straightforward relation between the I-V curves and the density of states and demonstrate conclusively that, under field, superconductivity is confined to the region near the "hills" resulting after the formation of the neck (fig.1).

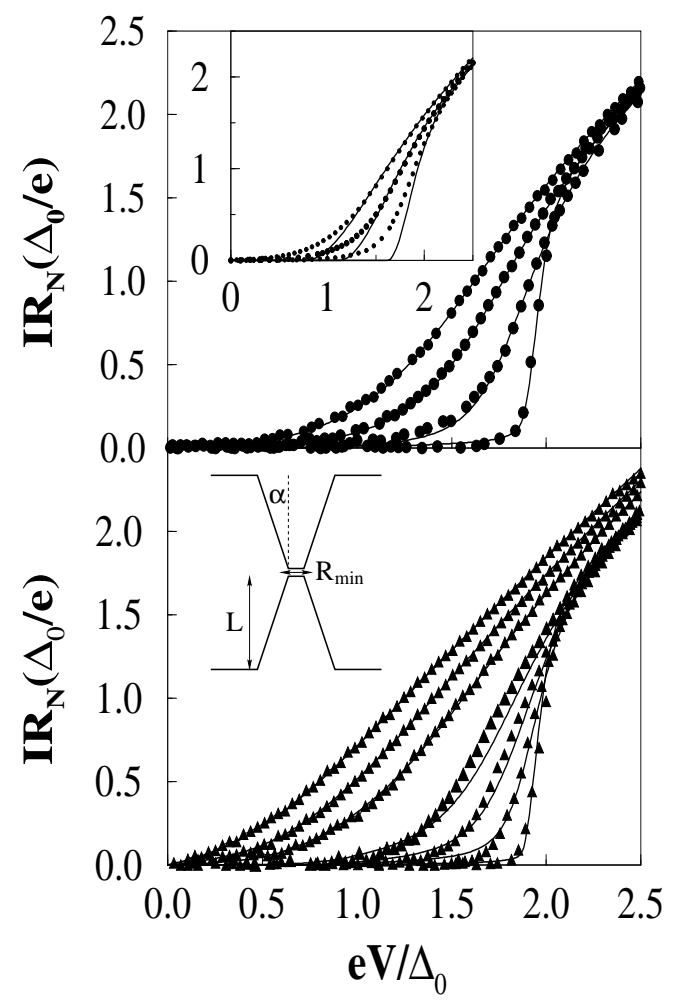

FIG. 3. Current-voltage characteristics, normalized to the tunneling resistance and the zero field gap respectively, for different fields applied parallel at $T=0.4 \mathrm{~K}$ in a) (from bottom to top $0,0.13,0.18$ and $0.23 \mathrm{~T}$ ) and at $T=1.5 \mathrm{~K}$ perpendicular to the axis of the neck (from bottom to top 0, 0.17, 0.34, $0.84,1.01,1.18 \mathrm{~T}$ ). Solid lines correspond to the fittings obtained with the geometry shown in the inset in b). For a) we use $R_{\min }=0.8 \xi, \alpha=56^{\circ}$ and $\xi=256 \AA$ And $L=2.9 \xi$, where $\xi$ is coherence length, and for b), $R_{\text {min }}=0.0, \alpha=27^{\circ}$, $\xi=270 \AA$ And $L=3.2 \xi$. Inset in a) shows the fittings (lines) to the finite magnetic field experimental curves in a) in (symbols) using an effective pair breaking parameter (from bottom to top: $\left.\Gamma_{0}=0.04,0.13,0.21\right)$.

This result demonstrates that it is possible to make
STM tips, which are superconducting even at magnetic fields as high as several tesla. The proximity effect of the parts of the tip that transit to the resistive phase at smaller fields needs to be taken into account in the calculations of their density of states.

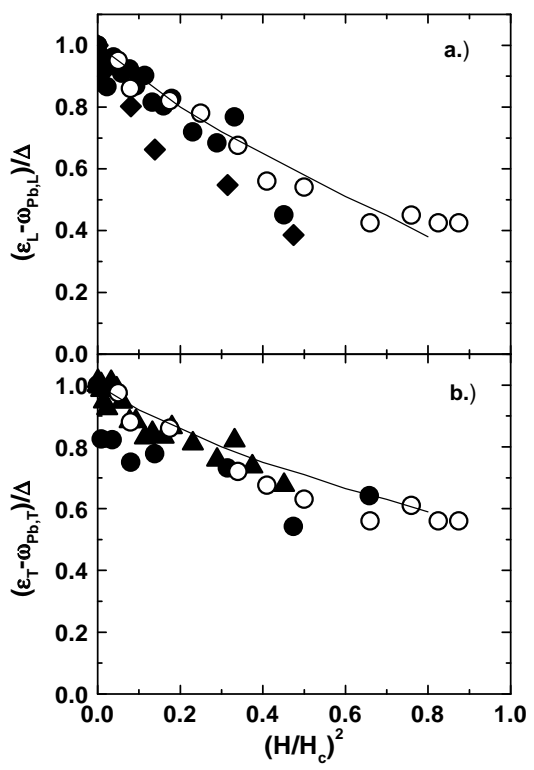

FIG. 4. The magnetic field behavior of the energy position $\epsilon_{L, T}$ of the features corresponding to transverse (index T, a.)) and longitudinal (index L, b.)) phonon energies, for the magnetic field applied parallel (closed circles) or perpendicular (closed triangles) to the neck. Superconductivity disappears respectively at $1.8 \mathrm{~T}$ and at $0.5 \mathrm{~T}$. Our data are compared to the result obtained using tunneling spectroscopy in thin films measured with the magnetic field parallel to the surface(open circles, see 23]), and to the theory of Ref. 24 (solid lines). To determine as accurately as possible the voltage position of the phonon modes as a function of the magnetic field, we make the mean value between positive and negative voltages and find $\epsilon_{L, T}$, the voltage position of the maximum in the second derivative $d^{2} I / d V^{2} 13$. For $\Delta$ we use two times the zero field gap value $2 \Delta_{0}$ in our data but $\Delta_{0}$ in the data of [23], where an N-S junction was used.

We now discuss the magnetic field behavior of features in the tunneling conductance associated to the phonon spectrum that appear at voltages above $2 \Delta$ (fig. $2 \mathrm{~b}$ ). Note that we have the unique possibility to follow these features during the confinement of superconductivity to a system of nanoscopic dimensions. Figure 4 shows the magnetic field behavior of the voltage position of the features in $d I / d V$ (fig. 2b). We plot $\left(\epsilon_{L, T}-\omega_{L, T}\right)$, normalized to $2 \Delta_{0}$, as a function of the squared magnetic field, normalized to the field for complete destruction of superconductivity $\left(H / H_{c}\right)^{2}$, together with the tunneling spectroscopy measurements in thin films published in [23,24]. The figure shows the variation of the voltage position of 
the phonon modes as a function of the magnetic field. It does not indicate changes in the phonon spectrum, but the decrease in the position in energy of the phonon modes in the superconducting density of states when the pair breaking effect of the field (and the proximity effect in our case) destroy the superconducting correlations. The calculation of the authors of Ref. 24 fits the experimental result within error (solid lines in Fig.4). It even reproduces the stronger decay in the higher energy longitudinal phonons, introducing pair breaking effects into the Eliashberg equations [24,27]. To make the same approach using a position dependent pair breaking parameter 20] is a formidable task which would require to solve self-consistently (both in energy and position) the Eliashberg equations [27]. The figure 4 shows, however, that the result is the same as in thin films and is compatible with the more commonly used pair breaking theory [22]. It demonstrates that the features related to strong coupling superconductivity are not sensitive to the precise form of the density of states, and it confirms previous work 23,24] about strong coupling superconductivity in the presence of pair breaking. Note that our experiment gives the additional check that the result is the same independent of the direction of the magnetic field or the geometry (data in thin films needed to be done with the magnetic field applied parallel to the surface).

In summary, we have examined superconducting connecting necks under magnetic fields and demonstrated the necessity of taking into account the proximity effect to explain their behavior. We clearly show that in nanofabricated STM tips made of type I materials, superconductivity is restricted to a nanoscopic region and remains at even high magnetic fields. This can considerably extend the application of STM as a probe. We have also demonstrated that in this system, the phonon structure of the density of states is not affected by the size reduction of the superconducting part.

We specially acknowledge discussions with J.T. Devreese, V.M. Fomin and W. Belzig and support from the ESF programme Vortex Matter in Superconductors at Extreme Scales and Conditions (VORTEX). We also acknowledge financial support from the the CICyT (Spain) through grants DGICYT PB97-0068, the Fermi liquid instabilities ESF programme (FERLIN) and the Comunidad Autónoma de Madrid. One of us (E.B.) also thanks financial support from the nsf-dmr0115947 and the Welch foundation.

* Grupo Intercentros de Bajas Temperaturas, Unidad Asociada ICMM-CSIC and LBT-UAM.

[1] See e.g. C.J. Chen, "Introduction to Scanning Tunnel- ing Microscopy", Oxford Series in Optical and Imaging Sciences, Oxford University Press (1993).

[2] N. Agraït, J.G. Rodrigo and S. Vieira, Phys. Rev. B, 48, 8499 (1993); J.G. Rodrigo, N. Agraït and S. Vieira, Phys. Rev. B, 50, 374 (1994).

[3] C. Untiedt, G. Rubio, S. Vieira and N. Agraï, Phys. Rev. B 56, 2154 (1997).

[4] J.R. Kirtley, J. of Mod. Phys. B, 4, 201 (1990).

[5] H.F. Hess, R.B. Robinson, J.V. Waszczak, Phys. Rev. Lett. 64, p. 2711 (1990).

[6] I. Maggio-Aprili, Ch. Renner, A. Erb, E. Walker, O. Fisher, Phys. Rev. Lett. 75, 2754 (1995).

[7] S.H. Pan, E.W. Hudson, K.M. Lang, H. Eisaki, S. Uchida, J.C. Davis, Nature 403, 746 (2000).

[8] G. Rubio-Bollinger, H. Suderow, S. Vieira, Physical Review Letters, 86, 5582 (2001).

[9] H. Suderow, E. Bascones, W. Belzig, F. Guinea, S. Vieira Europhysics Letters, 50, 749 (2000)

[10] H. Suderow, S. Vieira, Physics Letters A, 275, 299 (2000).

[11] M. Poza, E. Bascones, J. G. Rodrigo, N. Agraït, S. Vieira and F. Guinea, Phys. Rev. B 58, 11173 (1998).

[12] V.R. Misko, V.M. Fomin and J.T. Devreese, Phys. Rev. B, 64, 14517 (2001).

[13] E.L. Wolf, "Principles of Electron Tunneling Spectroscopy", Oxford University Press (1989).

[14] H. Suderow, S. Vieira, in preparation.

[15] R. Mersevey, Phys. Scr. 38, 272 (1988).

[16] S.H. Pan, E.W. Hudson, J.C. Davis, Applied Phys. Lett., 73, 2992 (1998).

[17] O. Naaman, W. Teizer, R.C. Dynes, Phys. Rev. Lett., 87, 97004 (2001); Rev. Sci. Instrum., 72, 1688 (2001).

[18] H. Suderow et al., to appear in Physica B.

[19] D.J. Scalapino, J.R. Schrieffer and J.W. Wilkins, Phys. Rev., 148, 263 (1966).

[20] The dependence of the critical field as a function of the form of the neck will be published in a future work (J.G. Rodrigo, H. Suderow, S. Vieira, in preparation).

[21] Provided that the field is applied parallel to the surface of the sample.

[22] K. Maki in "Superconductivity", vol. 2, R. D. Parks ed., M. Dekker (New York, 1969).

[23] Th.H.M. Rasing, H. W. M. Salemink, P. Wyder, S. Strassler, Phys. Rev. B, 23, 4470 (1981).

[24] J.M. Daams, H.G. Zarate, J.P. Carbotte, Phys. Rev. B, 30, 2577 (1984).

[25] J.S. Meyer, B.D. Simons, cond-mat/0111039 also discuss pair breaking in thin films beyond mean field.

[26] K. D. Usadel, Phys. Rev. Lett. 25, 507 (1970).

[27] G.M. Eliashberg, Zh. Eksperim. i Teor. Fiz. 38, 966 (1960)[Soviet. Phys. JETP 11, 696 (1960)]. 